\title{
Administration of Bovine Milk Oligosaccharide to Weaning Gnotobiotic Mice Inoculated with a Simplified Infant Type Microbiota
}

\author{
Louise Margrethe Arildsen Jakobsen ${ }^{1, * \mathbb{D}}$, Ulrik Kræmer Sundekilde ${ }^{1} \mathbb{D}$, Henrik Jørgen Andersen ${ }^{2}$, Witold Kot ${ }^{3}$, \\ Josue Leonardo Castro Mejia ${ }^{4}$, Dennis Sandris Nielsen ${ }^{4}\left(\mathbb{D}\right.$, Axel Kornerup Hansen ${ }^{5}$ (D) \\ and Hanne Christine Bertram ${ }^{1}$ (D)
}

Citation: Jakobsen, L.M.A.; Sundekilde, U.K.; Andersen, H.J.; Kot, W.; Mejia, J.L.C.; Nielsen, D.S.; Hansen, A.K.; Bertram, H.C. Administration of Bovine Milk Oligosaccharide to Weaning Gnotobiotic Mice Inoculated with a Simplified Infant Type Microbiota. Microorganisms 2021, 9, 1003. https://doi.org/10.3390/ microorganisms 9051003

Academic Editor: Seong-Tshool Hong

Received: 7 April 2021

Accepted: 4 May 2021

Published: 6 May 2021

Publisher's Note: MDPI stays neutral with regard to jurisdictional claims in published maps and institutional affiliations.

Copyright: (c) 2021 by the authors. Licensee MDPI, Basel, Switzerland. This article is an open access article distributed under the terms and conditions of the Creative Commons Attribution (CC BY) license (https:/ / creativecommons.org/licenses/by/ $4.0 /)$.
1 Department of Food Science, Aarhus University, Agro Food Park 48, 8200 Aarhus N, Denmark; uksundekilde@food.au.dk (U.K.S.); hannec.bertram@food.au.dk (H.C.B.)

2 Arla Food Ingredients P/S, Sønderhøj 10, 8260 Viby J, Denmark; hejan@arlafoods.com

3 Department of Plant and Environmental Sciences, University of Copenhagen, Thorvaldsensvej 40, 1871 Frederiksberg C, Denmark; wk@plen.ku.dk

4 Department of Food Science, University of Copenhagen, Rolighedsvej 30, 1958 Frederiksberg C, Denmark; jcame@food.ku.dk (J.L.C.M.); dn@food.ku.dk (D.S.N.)

5 Department of Veterinary and Animal Sciences, Faculty of Health and Medical Sciences, University of Copenhagen, Grønnegårdsvej 17, 1870 Frederiksberg C, Denmark; akh@sund.ku.dk

* Correspondence: loujak@food.au.dk; Tel.: +45-2073-1316

Abstract: Bovine milk oligosaccharides (BMO) share structural similarity to selected human milk oligosaccharides, which are natural prebiotics for infants. Thus, there is a potential in including BMOs as a prebiotic in infant formula. To examine the in vivo effect of BMO-supplementation on the infant gut microbiota, a BMO-rich diet $(2 \% w / w)$ was fed to gnotobiotic mice $(n=11)$ inoculated with an infant type co-culture and compared with gnotobiotic mice receiving a control diet $(n=9)$. Nuclear magnetic resonance metabolomics in combination with high-throughput 16S rRNA gene amplicon sequencing was used to compare metabolic activity and microbiota composition in different compartments of the lower gastrointestinal tract. BMO components were detected in cecum and colon contents, revealing that BMO was available for the gut bacteria. The gut microbiota was dominated by Enterobacteriaceae and minor abundance of Lactobacilliaceae, while colonization of Bifidobacteriaceae did not succeed. Apart from a lower E. coli population in cecum content and lower formate (in colon) and succinate (in colon and cecum) concentrations, BMO supplementation did not result in significant changes in microbiota composition nor metabolic activity. The present study corroborates the importance of the presence of bifidobacteria for obtaining microbial-derived effects of milk oligosaccharides in the gastrointestinal tract.

Keywords: infant nutrition; microbiome; NMR metabolomics; sialylated oligosaccharides; synthetic gut microbiota

\section{Introduction}

Human milk contains nutritional components for the growing infant and the microbiota of the infant. Around $15 \%$ of the energy in human breast milk is composed of complex carbohydrates, the so-called human milk oligosaccharides (HMOs). HMOs are nutritionally unavailable to the infant; however, they act as prebiotics that are either selectively metabolized by gut bacteria, whereby they become vital for establishment of a healthy microbiota in the infant [1], or they provide protection against infections through decoy of pathogens [2,3]. The first 1000 days (from conception until 2 years of age) of a child's life is an important period in regard to establishing the gut microbiota and is particularly important for the healthy development of the child [4]. During weaning (6-24 months 
postpartum), the child transitions from exclusive breast-feeding towards gradual introduction of solid foods. It appears that breast milk maintains a Bifidobacterium-rich gut microbiota as long as the child is partially breastfed, despite introduction of solid foods [4]. Infant formula is sometimes enriched with galacto-oligosaccharides to mimic some of the bifidogenic effects of oligosaccharides in human milk. Additionally, studies have shown beneficial effects of synthetic galacto-oligosaccharides and lactose in modulating the expression of mucus-related genes and ascribing this to a structure specific effect [5]. Another group of oligosaccharides in human colostrum and mature milk are the sialylated milk oligosaccharides, with $6^{\prime}$-sialyllactose and $3^{\prime}$-sialyllactose being the most abundant (up to 500 and $300 \mathrm{mg} / \mathrm{L}$, respectively) [6]. A higher content of sialylated oligosaccharides in breast milk from Malawian mothers appears to correlate positively with the growth of severely stunted infants and mouse and piglet studies have confirmed this effect to be mediated through an improved microbiota-dependent utilization of nutrients in the Malawian diets [7]. Bovine milk oligosaccharides (BMOs) represent a good source of sialylated oligosaccharides [8]. Since sialylated oligosaccharides are only present in trace amounts in infant formula (14-288 mg/L) [6], there is a potential for improving the bifidogenic effect of formula milk through enrichment with sialylated oligosaccharides. In a previous in vitro study, we found that $\mathrm{BMO}$ positively affects the composition of an infant type co-culture [9]. The infant type co-culture consisted of a total of eight bacteria representative of the infant gut microbiota, including strains of Bifidobacterium, Lactobacillus, Clostridium, Escherichia, Parabacteroides and Staphylococcus. The co-culture studies showed that BMO in combination with lactose or galactooligosaccharides resulted in a metabolic pattern characterized by low $\mathrm{pH}$ and high content of organic acids, particularly lactate. Furthermore, a combination of $\mathrm{BMO}$ and lactose proved to support growth of Bifidobacterium longum subsp. longum, while at the same time inhibiting growth of C. perfringens and E. coli [9]. Thus, there appears to be a potential benefit in introducing a bifidogenic effect by including $\mathrm{BMO}$ as a prebiotic ingredient in infant formula. The aim of the present study was to inoculate germ-free mice with an infant type co-culture to test the in vivo effect of a BMO-rich diet $(2 \% w / w)$ on the composition and metabolic activity of the microbiota.

\section{Materials and Methods}

\subsection{Experimental Diets}

The experimental diets were formulated by Research Diets (Research Diets, Inc. New Brunswick, NJ, USA). The BMO diet (BMO) contained bovine milk oligosaccharide enriched whey (Lacprodan SAL-10 ${ }^{\circledR}$, Arla Foods Ingredients Group P/S, Aarhus, Denmark) composed of $68 \%(w / w)$ lactose and $10 \%(w / w)$ oligosaccharides, including $4.5 \%$ sialyllactose, as well as other acidic and neutral bovine milk oligosaccharides (not quantified). The control diet $(\mathrm{CON})$ contained no $\mathrm{BMO}$ and the lactose content was controlled for by addition of a similar amount as present in the BMO diet $(14 / 100 \mathrm{~g})$. The nutritional composition of the two diets is given in Table 1.

Table 1. Nutritional composition of the BMO and CON diet.

\begin{tabular}{ccc}
\hline Per 100 g & BMO & CON \\
\hline Energy [Kcal] & 351 & 367 \\
Protein [g] & 15.8 & 16.6 \\
Carbohydrate [g] & 57.5 & 60.2 \\
Lactose [g] & 13.6 & 14.3 \\
Fat [g] & 6.4 & 6.7 \\
Fibre [g] & 8.8 & 9.3 \\
Oligosaccharide [g] & 2.0 & 0.0 \\
\hline
\end{tabular}

The diets were formulated with isocaloric macronutrient compositions: protein (18\%), carbohydrate (66\%) and fat (16\%). The source of fibers in the BMO diet was oligosaccharides 
$(2.0 / 100 \mathrm{~g})$ and cellulose $(6.8 / 100 \mathrm{~g})$, whereas in the CON diet the fibers purely consisted of cellulose $(9.3 / 100 \mathrm{~g})$.

The synthetic diets were packed in $500 \mathrm{~g}$ portions in heat-sealed bags under continuous nitrogen purge and the bags were subsequently irradiated twice.

\subsection{Animals}

Germ-free C57BL/6NTac mice (Taconic Biosciences, Ll. Skensved, Denmark) ( $n=26)$ were raised with the mother in a sterile environment until three weeks after birth. The mice were then moved to a separate isolator where they were weaned on a standard isolator irradiated diet (Altromin1314, Brogaarden, Lynge, Denmark) for 1 week until inoculation with the 8-strain infant type co-culture. On the day of inoculation, the mice were randomly assigned to either experimental (BMO, $n=13)$ or control groups $(\mathrm{CON}, n=13)$ and received one of the synthetic diets for two weeks (Figure 1).

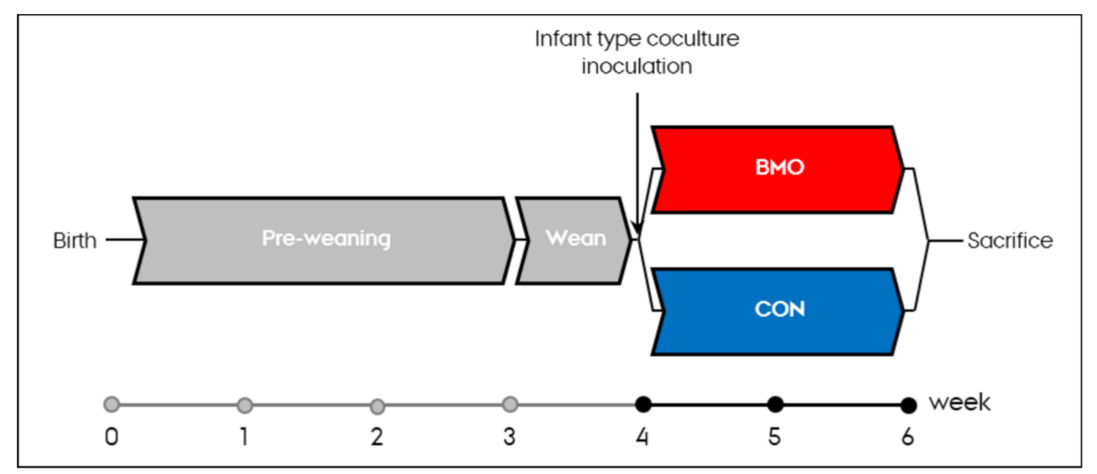

Figure 1. Study design of the gnotobiotic mouse study showing the time from birth, pre-weaning, weaning, experimental period until sacrifice. BMO: synthetic diet containing $2 \%(w / w)$ bovine milk oligosaccharides. CON: synthetic control diet.

\subsection{Ethics Statement}

The experiment was approved by the Animal Experimentation Committee under the Danish Food Administration and was licensed according to the Danish Animal Experimentation Act and the EU Directive 2010/63/EU (License No 2017-15-0201-01262).

\subsection{Infant Type Co-Culture for Inoculation}

The infant type co-culture was composed of eight bacterial type strains: Bifidobacterium longum subsp. longum (DSM 20219), Bifidobacterium breve (DSM 20213), Lacticaseibacillus rhamnosus (LMG 18243), Staphylococcus aureus (DSM 20231), Staphylococcus epidermidis (DSM 20044), Clostridium perfringens (DSM 756), Parabacteroides distasonis (DSM 20701) and Escherichia coli (DSM 30083). The eight bacteria were activated from frozen stocks into fresh optimal media as described previously [9] and $200 \mu \mathrm{L}$ aliquots of co-culture containing approximately $10^{8} \mathrm{CFU}$ of each strain were kept frozen until use. The co-culture was administered to the mice by holding them loosely in the neck skin and administering the entire content of one aliquot to the mouth using a pipette.

\subsection{Sample Collection}

Fecal samples were collected from the mice three days after weaning, halfway through the second week of intervention and on the day of sacrifice. In addition, fecal samples were collected from the cages before inoculation and on the day of sacrifice to check sterility. Consumption of the isolator diet and the experimental diets were registered for each cage weekly using a spring scale (Pesola Micro Line, Capacity $=30 \mathrm{~g}$, Division $=0.25 \mathrm{~g}$, Switzerland). Final body weight of the animals was registered on the day of sacrifice.

Mice were sacrificed and blood was sampled into EDTA-coated tubes and plasma obtained by centrifugation at $8000 \mathrm{~g}$ for $10 \mathrm{~min}$. From the gastrointestinal tract, content 
of the cecum and proximal colon was sampled into individual tubes and snap frozen in liquid nitrogen. All samples were stored at $-80^{\circ} \mathrm{C}$ until further analysis.

\subsection{Sample Preparation for ${ }^{1} H$ Nuclear Magnetic Resonance (NMR) Spectroscopy}

Plasma samples were thawed at room temperature and then filtered through prewashed $0.5 \mathrm{~mL}$ 10k Millipore centrifugal filter units (Amicon Ultra, Merck Millipore Ltd., Billerica, MA, USA) by centrifugation at $4{ }^{\circ} \mathrm{C}$ at $10,000 \mathrm{~g}$ for $1 \mathrm{~h}$. A volume of $150 \mu \mathrm{L}$ plasma filtrate was transferred to a $5 \mathrm{~mm} \mathrm{NMR}$ tube with $300 \mu \mathrm{L} \mathrm{D}_{2} \mathrm{O}$ (deuterium oxide, $99.9 \%$, Cambridge Isotope Laboratories, Andover, MA, USA) and $100 \mu \mathrm{L} \mathrm{D}_{2} \mathrm{O}$ containing $0.05 \%$ $(w / v)$ 3-(trimethylsilyl)-2,2,3,3-tetradeuteropropanoic acid (TSP, Sigma-Aldrich, St. Louis, $\mathrm{MO}, \mathrm{USA})$.

Cecal content samples were thawed at room temperature and $~ 100 \mathrm{mg}$ sample material was weighed out. A volume of $800 \mu \mathrm{L}$ distilled $\mathrm{H}_{2} \mathrm{O}$ was added to achieve an approximate $\mathrm{W}$ (sample): $\mathrm{V}\left(\mathrm{H}_{2} \mathrm{O}\right)$ ratio of 1:9 for extraction of metabolites. The sample was vortexed for $10 \mathrm{~s}$ and centrifuged at $4{ }^{\circ} \mathrm{C}$ at $14,000 \times g$ for $5 \mathrm{~min}$. The supernatant was filtered through $0.5 \mathrm{~mL}$ 10k Millipore centrifugal filter units by centrifugation at $4{ }^{\circ} \mathrm{C}$ at $14.000 \times g$ for $30 \mathrm{~min}$ and the filtering procedure repeated until a total volume of $400 \mu \mathrm{L}$ of filtered supernatant could be obtained. The pooled supernatant was added to $5 \mathrm{~mm}$ NMR tube with $50 \mu \mathrm{L}$ phosphate buffer (pH 7.4, 0.6 M) containing internal standard $10 \mathrm{mM}$ DSS (3(trimethylsilyl)-1-propanesulfonic acid- $\mathrm{d}_{6}$ sodium salt (Sigma-Aldrich, St. Louis, MO, USA) and $200 \mu \mathrm{L} \mathrm{D}_{2} \mathrm{O}$. The $\mathrm{pH}$ was measured using a $\mathrm{pH}$ meter fitted with a silver electrode (Radiometer, Copenhagen, Denmark) before addition of buffer.

Colon content samples were thawed at room temperature and 15-50 mg of sample material was weighed out. A volume of distilled $\mathrm{H}_{2} \mathrm{O}$ corresponding to a $\mathrm{W}$ (sample): $\mathrm{V}$ $\left(\mathrm{H}_{2} \mathrm{O}\right)$ ratio of 1:20 was added to the sample, it was vortexed twice for 10-20 s to dissolve the pellet and centrifuged at $1000 \times g$ for $1 \mathrm{~min}$. The supernatant was filtered through $10 \mathrm{k}$ Millipore centrifugal filter units at $10,000 \times g$ for $30 \mathrm{~min}$ and repeated until a volume of $400 \mu \mathrm{L}$ filtrate was obtained. The collected supernatant was added to $5 \mathrm{~mm}$ NMR tubes with $100 \mu \mathrm{L}$ phosphate buffer ( $\mathrm{pH} 7.4,0.6 \mathrm{M}$ ) containing $10 \mathrm{mM}$ DSS and $100 \mu \mathrm{L} \mathrm{D}_{2} \mathrm{O}$.

\section{7. ${ }^{1} \mathrm{H}$ NMR Spectroscopy and Metabolomics}

For plasma, cecal and colon content samples, NMR spectra were acquired on a Bruker Avance III $600 \mathrm{MHz}$ NMR spectrometer (Bruker BioSpin, Gmbh, Rheinstetten, Germany) operating at a proton NMR frequency of $600.13 \mathrm{MHz}$ for ${ }^{1} \mathrm{H}$ and equipped with a $5 \mathrm{~mm}$ TXI probe. The 1D NOESY pulse experiment with presaturation of the spectral region containing the water peak (noesypr1d) was used with a recycle delay of $5 \mathrm{~s}$. A total of 128 FIDs were acquired and the acquisition parameters included 32K complex data points, a spectral width of $7289 \mathrm{~Hz}(12.15 \mathrm{ppm})$ and an acquisition time of $2.25 \mathrm{~s}$. The measurements were performed at a temperature of $298 \mathrm{~K}\left(25^{\circ} \mathrm{C}\right)$. NMR spectra were automatically baseline corrected and manually phase corrected using Topspin (Version 3.0, Bruker BioSpin).

In Matlab (R2016, MathWorks Inc., Natick, MA, USA), processed NMR spectral files were aligned by icoshift [10] and calibrated to the TSP or DSS resonance at $0.0 \mathrm{ppm}$. Spectral regions with no or disturbing resonances were trimmed, by excluding the regions containing the ethanol resonances at 3.67-3.63 and 1.26-1.12 ppm, the water resonance at 4.90-4.75 ppm and finally the regions above $10.0 \mathrm{ppm}$ and below $0.5 \mathrm{ppm}$. Finally, the area of the NMR spectra were normalized to the area of TSP or DSS and binned into $0.005 \mathrm{ppm}$ intervals in order to reduce the amount of data points.

For multivariate analysis the pre-processed data were loaded into SIMCA (15, Sartorius Stedim Data Analysis AB), pareto-scaled and principal component analysis (PCA) performed to visualize the variation in the data. If PCA models showed clear separation between diet groups, orthogonal, partial least squares discriminative analysis (OPLSDA) was performed using diet as Y-variable to maximize the separation between dietary groups and S-line plots were calculated to identify the bins contributing the most to group discrimination. Full cross-validation was performed using the leave-one-out method to 
estimate model validity $\left(\mathrm{Q}^{2}\right)$. Metabolite identification and quantification was performed in Chenomx NMR suite (Chenomx Inc., Edmonton, Alberta, Canada) using the build-in $600 \mathrm{MHz}$ library.

\subsection{DNA Extraction}

DNA was extracted from cecal content and fecal samples using the Bead-Beat Micro AX Gravity kit with mechanical lysis (A\&A Biotechnology, Gdańsk, Poland) according to the manufacturer's protocol with a few modifications. Briefly, cecal or fecal material was added to the bead-beat tubes, approximately $100 \mathrm{mg}$ per tube. A reaction mixture containing $1 \mathrm{~mL}$ LSU buffer, $10 \mu \mathrm{L}$ lysozyme and $5 \mu \mathrm{L}$ mutanolysin was added to each tube, followed by vortexing and then incubation at $50{ }^{\circ} \mathrm{C}$ for $20 \mathrm{~min}$. A volume of $20 \mu \mathrm{L}$ Proteinase $\mathrm{K}$ was then added, followed by bead beating in a FastPrep-24 5G instrument (MP Biomedicals, Santa Ana, CA, USA) in three cycles of $20 \mathrm{~s}$ at a speed of $6.5 \mathrm{M} / \mathrm{s}$. The samples were then incubated at $50{ }^{\circ} \mathrm{C}$ for $20 \mathrm{~min}$ and the remaining steps performed according to the manufacturer's protocol. A NanoDrop spectrometer (Thermo Fisher Scientific, Wilmington, DE, USA) was used to determine the yield and purity of DNA. The yield was ranging $29-181 \mathrm{ng} / \mu \mathrm{L}$ and purity $\left(\mathrm{A}_{260 / 280}\right)$ was $1.8-2.1$ for all samples. After extraction, the DNA was stored at $-60^{\circ} \mathrm{C}$ until further processing.

\subsection{High-Throughput $16 \mathrm{~S}$ rRNA Gene Amplicon Sequencing}

High throughput $16 \mathrm{~S}$ rRNA gene amplicon sequencing was conducted on DNA from cecum content and fecal samples that were collected during the experimental period and at sacrifice to determine the microbiota composition. The primers, NXt_388_F ( $5^{\prime}$ TCGTCGGCAGCGTCAGATGTGTATAAGAGACAGACWCCTACGGGWGGCAGCAG-3') and NXt_518_R (5'-GTCTCGTGGGCTCGGAGATGTGTATAAGAGACAGATTACC GCGG CTGCTGG-3') were designed with adapters Nextera Index $\mathrm{Kit}^{\circledR}$ (Illumina, CA, USA) and targeted the V3 region ( 190 bp). Amplicon library preparation, purification and sequencing was performed similar as described by Castro-Mejía [11]. Briefly, purification of the amplified fragments was performed by AMPure XP Beads (Beckman Coulter Genomic, CA, USA) and prior to library pooling, the clean constructs were quantified using a Qubit Flourometer (Invitrogen, Carslbad, CA, USA) and mixed in approximately equal concentrations to ensure even representation of reads per sample. Finally, $2 \times 150$ cycles, pair-ended Illumina NextSeq sequencing using the Mid Output v2 chemistry (Illumina, CA, USA) was performed. High-throughput sequencing data were processed as previously described [11]. Briefly, the raw data containing pair-ended reads were merged and trimmed as described previously [12]. Zero radius operational taxonomic units (zOTUs) were conducted using the UNOISE pipeline [13]. The green genes (v13.8) 16S rRNA gene collection was used as a reference database [14]. Reads from OTU tables were aggregated on the family level, which was the most robust level of discriminating the bacteria in the infant type coculture. Information on genus was investigated if relevant to the interpretation of results. Sequences assigned to OTU $\mathrm{f}$ _S Streptococcaeceae; $\mathrm{g}$ _LLactococcus were filtered out as they originated from Lactococcus precipitated casein in the diet [15]. Samples with low DNA concentration $(<0.1 \mathrm{ng} / \mu \mathrm{L})$ were excluded from the abundance analysis. The R software (version 3.6.1, The R Foundation, Vienna, Austria) was used for calculating and visualizing the relative abundances constituting more than $2 \%$ of the total relative abundance.

\subsection{Real Time Quantitative Polymerase Chain Reaction (RT qPCR)}

A SYBR ${ }^{\mathrm{TM}}$ Green assay using primers designed to target the non-conserved regions of the 16S rRNA gene of selected bacterial species was set-up. The primers are described elsewhere [9]. The bacterial DNA from pure cultures was serially diluted in RNase free water $\left(10^{-1}\right.$ to $\left.10^{-6}\right)$, while DNA from cecal samples was analyzed without dilution. One master mix per primer set was prepared consisting of Fast SYBR ${ }^{\circledR}$ Green I Master Mix, primer mix (forward and reverse diluted to $5 \mu \mathrm{M}$ ) and RNAse free water. Five $\mu \mathrm{L}$ DNA or RNAse free water for non-template controls (NTC) was added to reach a final reaction 
volume of $20 \mu \mathrm{L}$. Two technical replicates were included per biological sample. The RT qPCR assay was run on a 7500 Fast Real-Time PCR System (Life Technologies ${ }^{\mathrm{TM}}$, Dublin, Ireland) using the following program: $50{ }^{\circ} \mathrm{C}(2 \mathrm{~min})$, at $95{ }^{\circ} \mathrm{C}(2 \mathrm{~min})$, forty cycles of: $95^{\circ} \mathrm{C}(15 \mathrm{~s}), 55^{\circ} \mathrm{C}(15 \mathrm{~s})$ and $72{ }^{\circ} \mathrm{C}(1 \mathrm{~min})$. Melting curve analysis was then run at $95{ }^{\circ} \mathrm{C}$ (15 s), $56{ }^{\circ} \mathrm{C}(1 \mathrm{~min}), 95{ }^{\circ} \mathrm{C}(30 \mathrm{~s})$ and $56{ }^{\circ} \mathrm{C}(15 \mathrm{~s})$ to determine the melt temperature of the amplicon.

\subsection{Statistical Analysis}

ANOVA was applied to test the effect of treatment group (BMO or CON diet) on feed intake, weight of mice at the end of intervention as well as treatment effect on metabolite concentrations in cecum content, colon content and plasma. Before ANOVA analysis, the datasets were checked for variance homogeneity and normality by visual inspection of residual vs. fitted and QQplots, respectively. Deviating samples were only removed if differed substantially from the rest of the samples of the same treatment, or if the sample contributed to non-normality of the dataset and an underlying reason for removal of the sample could be identified. Data analysis and visualization was performed in $\mathrm{R}$ (version 3.6.1, The R Foundation, Vienna, Austria).

\section{Results}

During the experimental period, three mice died, one of which were fed the BMO diet and two who were fed the CON diet. Unfortunately, additional mice showed contamination of fecal or cecum samples and were therefore excluded from all analyses. The final distribution of mice on each diet were $\mathrm{BMO}(n=11)$ and $\mathrm{CON}(n=9)$. Consumption of the $\mathrm{BMO}$ diet was higher than $\mathrm{CON}$ diet at week 1 (Figure $2 \mathrm{~A}, p=0.037$ ) and in total over the two weeks (Figure 2C, $p=0.028$ ), but the final body weight of the mice in each treatment group was not significantly different (Figure 2D, $p=0.83$ ).

(A)

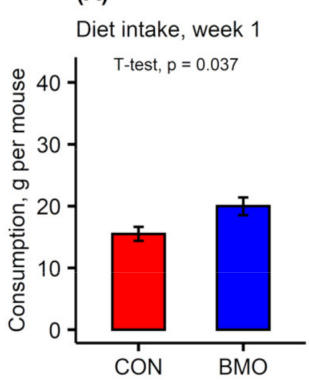

(B)

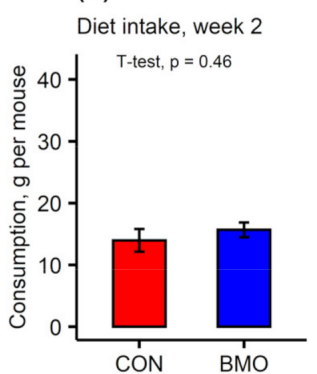

(C)

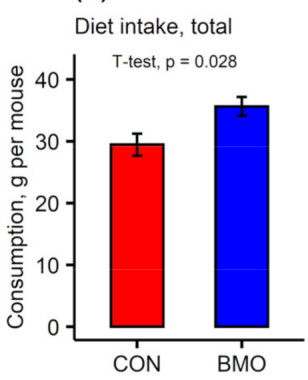

(D)

Weight of mice, sacrifice

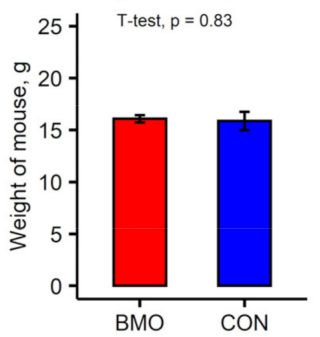

Figure 2. Background data on mice. (A-C) Diet consumption during week 1 and 2 and in total over the course of the experimental period was measured per cage and was normalized to the number of animals in individual cages to express $\mathrm{g} /$ mouse and (D) Body weight of mice at the end of the experimental period (BMO: $n=11, \mathrm{CON}: n=9$ ). Values are means and error bars show standard deviation.

\subsection{Microbiota Composition during Experimental Period}

Only two of the eight strains in the infant type coculture were identified on the family level. The microbiota composition during the experimental period (Figure 3) was dominated by the families Enterobacteriaceae and Lactobacilliaceae, which correspond to the families of E. coli and L. rhamnosus of the infant type co-culture, respectively. Collectively, the data did not indicate differential microbiota response between the two treatments after 7 days of intervention; the average relative abundance of Enterobacteriaceae was $89 \%$ $( \pm 6 \%)$ and $86 \%( \pm 10 \%)$ and Lactobacilliaceae constituted $11( \pm 6 \%)$ and $14 \%( \pm 10 \%)$ in $\mathrm{BMO}$ and $\mathrm{CON}$ treatments, respectively. Intriguingly, the other bacterial families that constituted the infant type co-culture were not observed in these fecal samples taken 7 days after inoculation. 


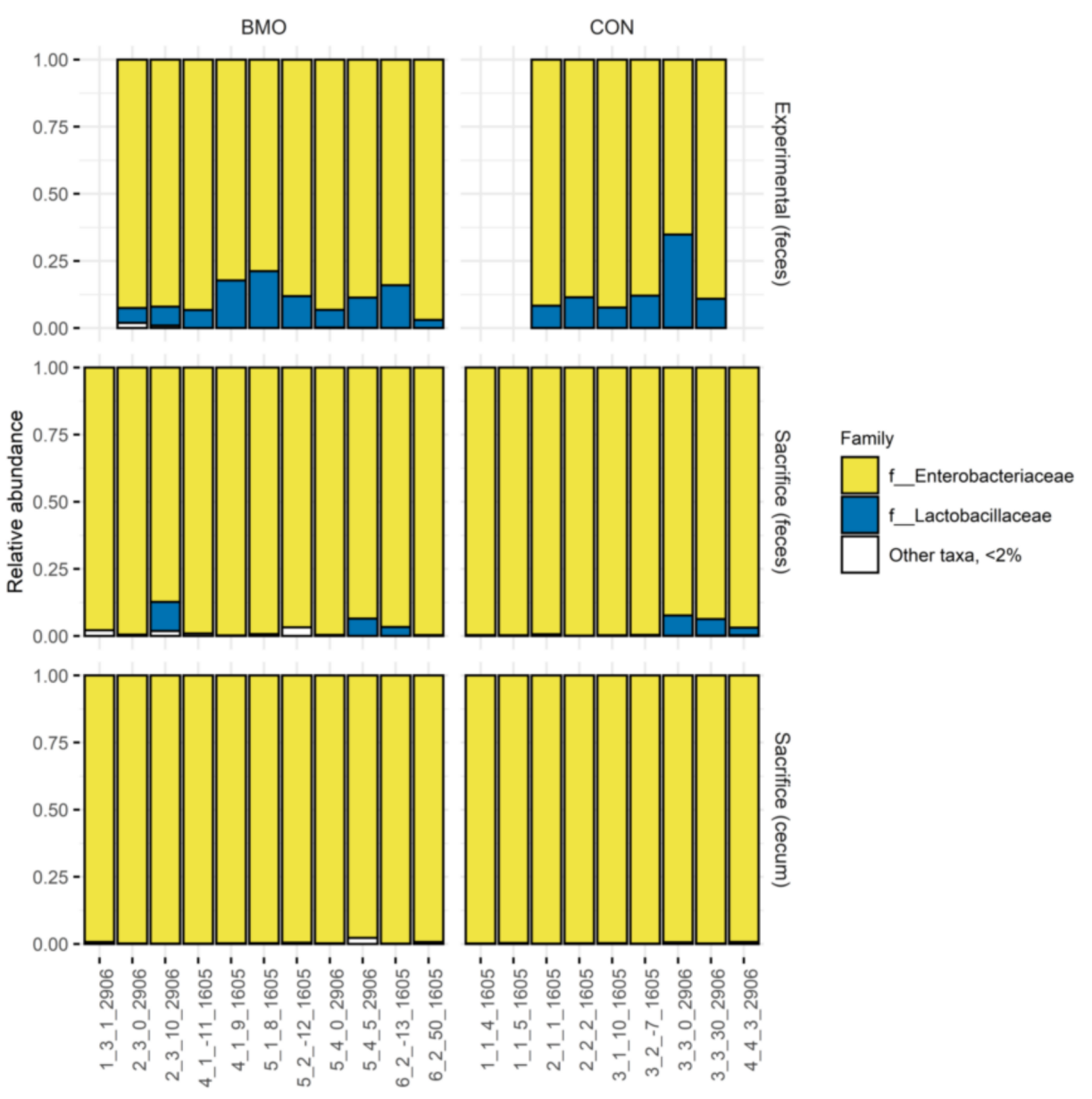

Figure 3. Relative abundance at family level ( $<2 \%$ relative abundance), horizontally grouped according to dietary treatment and vertically grouped according to sampling time (experimental or sacrifice) and sample type (feces or cecum content). Each vertical bar with a unique number represents samples from an individual mouse. Sequencing data of fecal samples from the experimental period was available from a total of 16 mice (BMO; $n=10, \mathrm{CON}: n=6)$, while for at the time of sacrifice, sequence data were available fecal and cecum content for a total of 20 mice (BMO: $n=11, \mathrm{CON}: n=9$ ) BMO: synthetic diet with $2 \%$ bovine milk oligosaccharides, CON: synthetic control diet with lactose.

\subsection{Microbiota Composition and Metabolites in Colon}

Due to limited sample material, it was not possible to compare the microbiota composition and metabolite profile of colon content one-to-one. Therefore, microbiome composition was performed on freshly collected fecal samples from each individual mouse before sacrifice and NMR metabolomics analyses were performed on proximal colon content after sacrifice. The fecal samples obtained at sacrifice showed a high abundance of Enterobacteriaceae and minor abundance of Lactobacilliaceae (Figure 3, mid panel), while other families constituting the infant type co-culture were not detected. Specifically, the relative abundance of Enterobacteriaceae was $97 \%( \pm 4 \%)$ in the BMO group and $98 \%( \pm 3 \%)$ in the CON group, while Lactobacilliaceae constituted $2 \%( \pm 4 \%)$ in the BMO group and $2 \%( \pm 3 \%)$ in the CON group. NMR metabolomics analysis showed a clear separation between the two treatment groups and a valid OPLS-DA model was obtained (Figure 4A, Q2 $=0.903$ ). Resonances that were enhanced with the BMO treatment included 3'-SL (the main sialyllactose in the $\mathrm{BMO}$ product), 4-hydroxyphenyl acetate and other $\mathrm{BMO}$ resonances around $8.5 \mathrm{ppm}$, while the resonance from succinate was enhanced with the CON treatment. Examination of the NMR spectra did not reveal resonances from lactose in colon content of mice from either of the dietary treatments (data not shown). 
(A) Sacrifice (colon content)

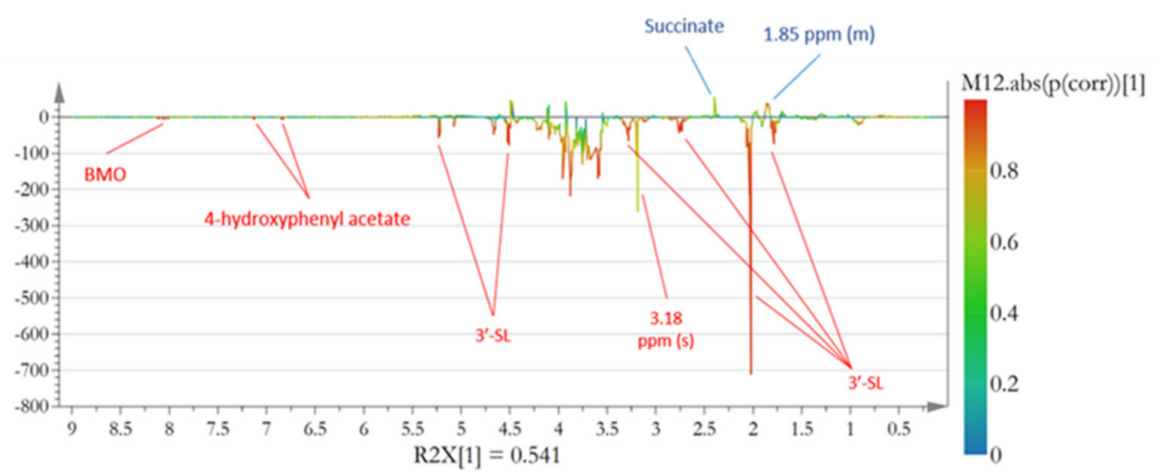

(B) Sacrifice (cecum content)

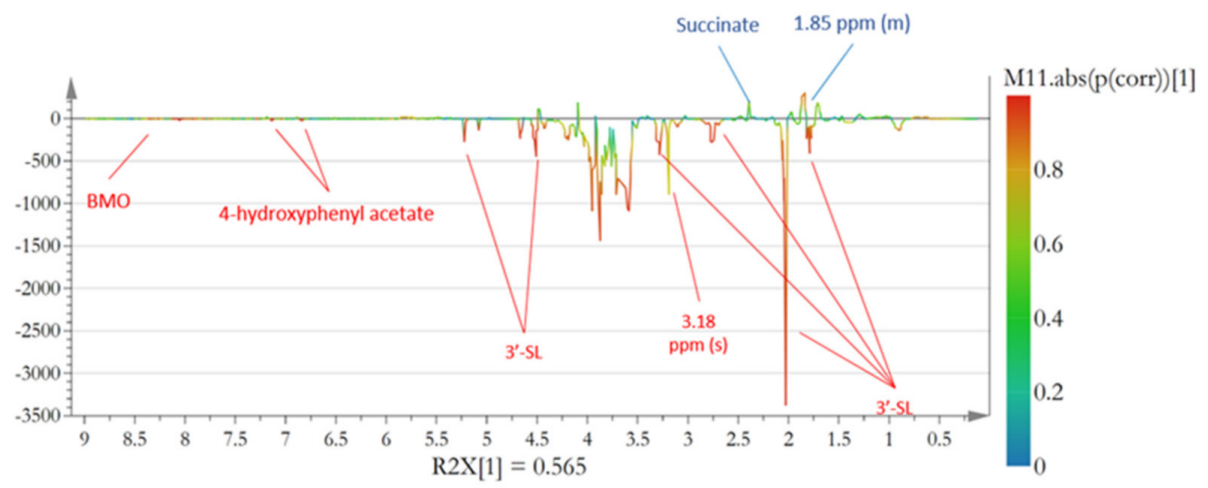

Figure 4. S-line plot from OPLS-DA showing ${ }^{1} \mathrm{H}$ NMR metabolomics data from samples collected at sacrifice. Positive resonances are correlated to $\mathrm{CON}$ treatment and negative resonances are correlated to BMO treatment. Color of the line indicates the significance of correlation. The OPLS-DA model validated using full-cross validation (leave-one-out) and each included one predictive and one orthogonal component, (A) Colon content, (Q2-value = 0.903), (B) Cecum content, (Q2 = 0.918).

Univariate statistical analyses revealed that the concentration of formate was higher in the CON treatment $(p=0.05)$ and so was succinate $(p=0.019)$, but the concentrations of SCFAs and other organic acids were not significantly different between the two treatment groups (Figure 5A).

\subsection{Microbiota Composition and Metabolites in Cecum Content}

Cecum content was sampled after sacrifice and divided into two aliquots: one for DNA extraction (16S rRNA gene amplicon sequencing and RT qPCR) and one for 1H NMR metabolomics. This allowed for a direct comparison between microbiota composition and metabolic pattern. The microbiota data (Figure 3, lower panel) showed that the cecum content was dominated by Enterobacteriaceae. The relative abundance of Enterobacteriaceae was above $99 \%$ in both $\mathrm{BMO}$ and CON treatments. Lactobacilliaceae were also present, but the relative abundance was lower than the $2 \%$ cut-off value. RT qPCR was performed to determine the absolute quantity of the two strains using primers specific for L. rhamnosus and $E$. coli. The results showed that $L$. rhamnosus was not significantly different between cecum samples from BMO and CON treatments $(1.8 \pm 1.3$ and $2.3 \pm 0.8 \log \mathrm{CFU} / \mathrm{g}$ cecum content, $p=0.2$ ). The absolute quantity of $E$. coli, however, was significantly lower in BMO ( $7.9 \pm 1.3 \log \mathrm{CFU} / \mathrm{g}$ cecum content) compared to $\mathrm{CON}(9.5 \pm 2.1 \mathrm{log} \mathrm{CFU} / \mathrm{g}$ cecum content) treatment $(p=0.036)$. Multivariate analysis of the ${ }^{1} \mathrm{H}$ NMR spectra showed a clear separation between the two dietary groups when employing OPLS-DA (Figure 4B, Q2 = 0.918). Resonances that were positively associated with BMO treatment were assigned to $3^{\prime} \mathrm{SL}$, 4-hydroxyphenyl acetate and other BMO resonances at $8.0 \mathrm{ppm}$. The CON treatment was 
associated with higher intensities of succinate and an unassigned multiplet at $1.85 \mathrm{ppm}$. Examination of the NMR spectra did not reveal resonances from lactose in cecum of mice from either of the dietary treatments (data not shown). Succinate was significantly higher in the CON treatment $(p=0.013)$, while isobutyrate tended to be higher $(p=0.061)$. The remaining SCFA and other organic acids did not reach statistical significance (Figure 5B).

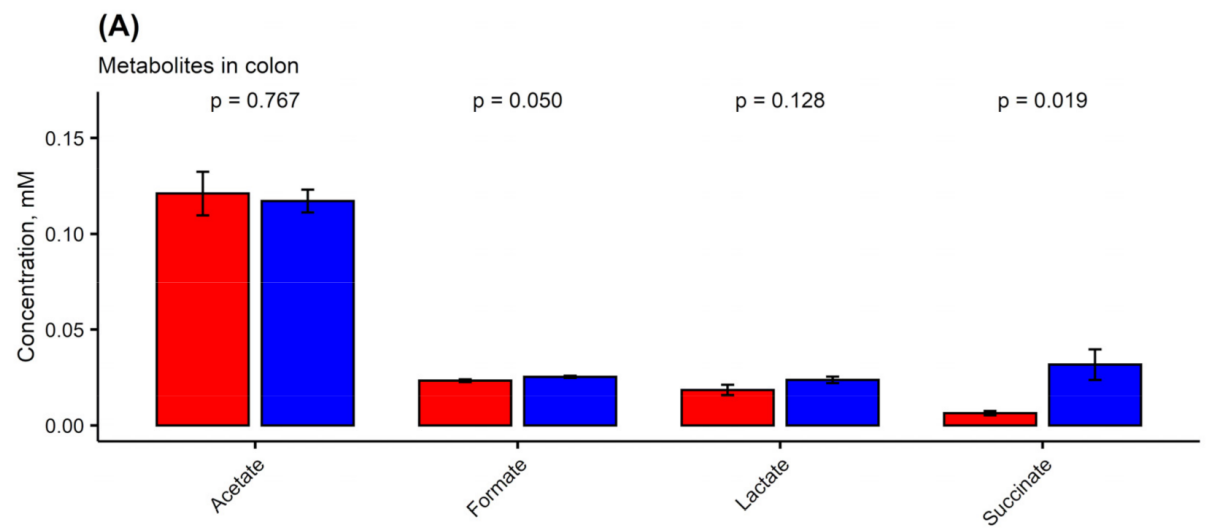

(B)

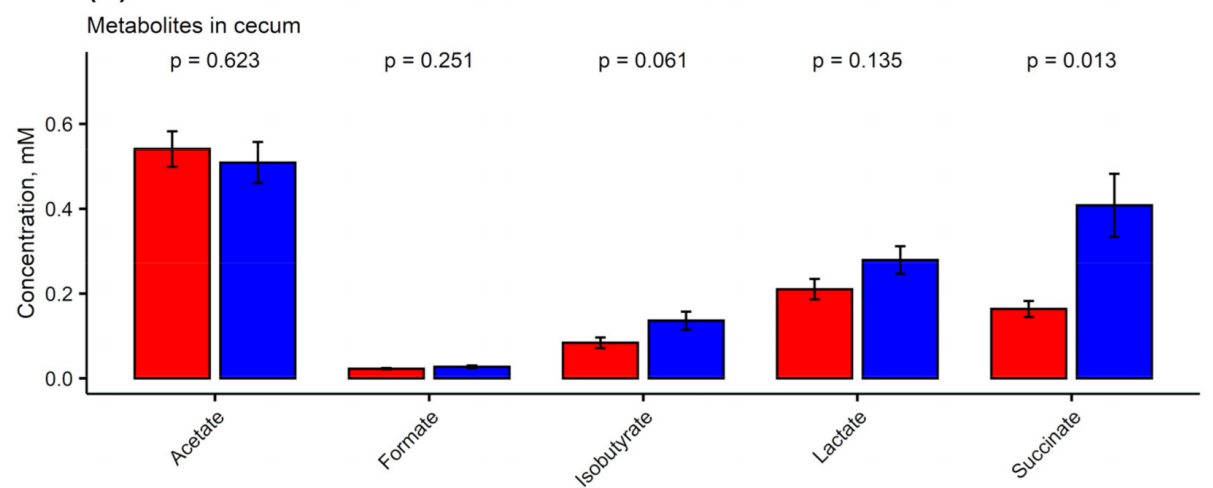

(C)

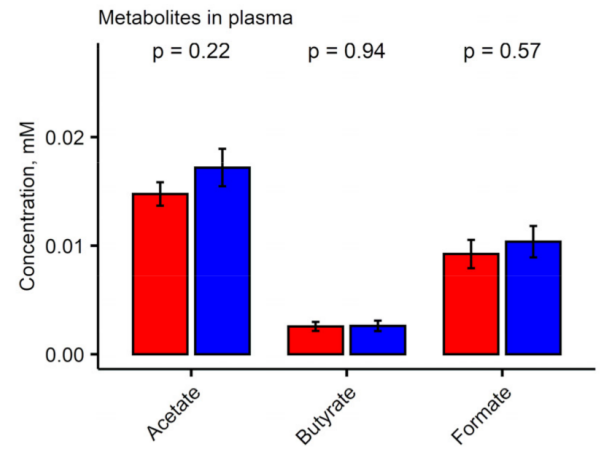

(D)

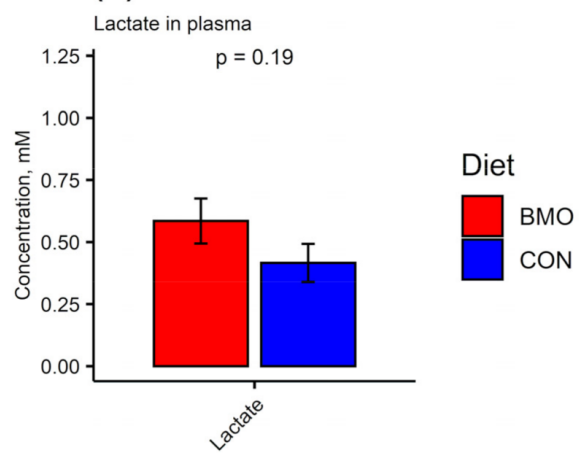

Figure 5. Quantified metabolites at sacrifice in (A) colon content (BMO: $n=7, \mathrm{CON}: n=7)$, (B) cecum content (BMO: $n=11, \mathrm{CON}: n=8),(\mathbf{C})$ metabolites in plasma and (D) lactate in plasma (BMO: $n=11$, CON: $n=8) . p$-values indicate significance level t-test on effect of diet on metabolite concentration. BMO: diet with $2 \%$ bovine milk oliogsaccharides, $\mathrm{CON}$ : control diet with lactose. Values are means and error bars show standard deviation.

\subsection{Relative Abundance of Enterobacteriaceae and Lactobacilliaceae at Weaning, Experimental} Period and Sacrifice

The abundance of Enterobacteriaceae and Lactobacilliaceae showed major variation at different time points and in different sample types (colon or cecum) in the study (Figure 6). The abundance of Lactobacilliaceae peaked in feces during the experimental period, decreased slightly in feces collected at sacrifice and was only present in very low abundance in cecum 
content collected at sacrifice (Figure 5A). Enterobacteriaceae increased after weaning to a point where this family constituted almost the entire population in cecum at sacrifice (Figure 6A).

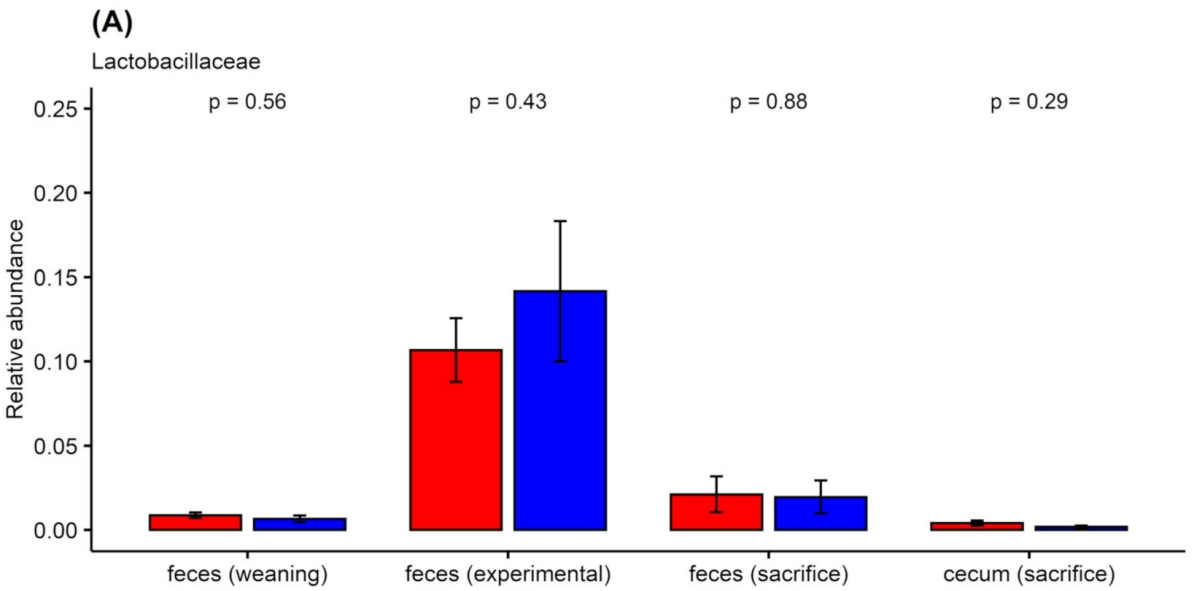

(B)

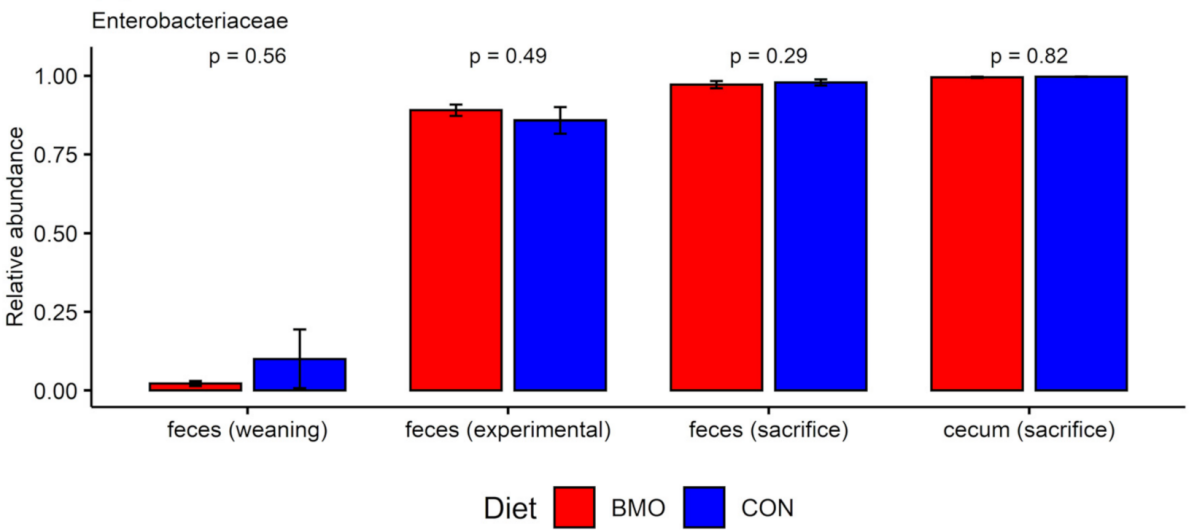

Figure 6. Relative abundance of selected families in samples collected throughout the study. (A) Lactobacilliaceae, (B) Enterobacilliaceae. The x-labels indicate the sample type and time of collection: feces (weaning): feces collected during weaning ( $\mathrm{BMO} n=5, \mathrm{CON} n=4$ ), feces (experimental): feces collected midway through the experimental period ( $\mathrm{BMO} n=10, \mathrm{CON} n=6$ ), feces (sacrifice): feces collected before sacrifice (BMO $n=11, \mathrm{CON} n=9$ ), cecum (sacrifice): cecum content collected after sacrifice (BMO $n=11, \mathrm{CON} n=9$ ). Colors indicate the dietary treatment: BMO: synthetic diet with $2 \%$ bovine milk oligosaccharide, CON: control diet with lactose. Dots show individual samples, bars are mean values and error bars show standard error. $p$-values above bars are $t$-test comparing the average relative abundance in samples from the two diets collected at the same time point.

\subsection{Metabolites in Plasma}

Acetate, butyrate, formate and lactate were quantified in the blood plasma samples collected on the day of sacrifice (Figure 5C). High concentrations of lactate were found in both dietary groups. None of the metabolites were significantly different between the two dietary groups.

\section{Discussion}

In the present study, germ-free weaning mice were inoculated with an infant type co-culture to produce a gnotobiotic mouse model for studying the in vivo effect of BMO supplementation on microbiota composition, metabolic activity in the lower gastrointestinal tract and alterations in plasma metabolites. Gnotobiotic mouse studies allow for the investigation of in vivo effects of, e.g., prebiotics in a human-like microbiota and provide unique prospects for confirming associations and proving causality in micro- 
biota research [16]. However, a key challenge with gnotobiotic mice studies relates to the successful colonization of animals with a microbiota that is not co-evolved with the host.

In the present study, feeding of either a BMO diet containing $2 \%$ bovine milk oligosaccharides or a control (CON) diet with lactose for two weeks resulted in fecal samples and a cecum content highly dominated by Enterobacteriaceae and Lactobacilliaceae. In contrast, none of the other strains included in the infant type co-culture were detected using the high-throughput $16 \mathrm{~S}$ rRNA gene amplicon sequencing approach. A previous study with infant type co-culture inoculation showed successful colonization of germ-free mice after administering a single gavage of infant type co-culture [17]. Following the infant type co-culture inoculation, the mice were fed a natural ingredient chow diet for four weeks and at the end of the experiment, the mice had a microbiota dominated by Enterobacteria, Bacteroides and Clostridium [17]. Interestingly, Martin et al. (2007) also identified bifidobacteria in the feces at the end of the experiment, which is in contrast, to the present study. Although the mode of inoculation was similar to Martin et al. (2007), the diets were different. In the present study, synthetic diets were formulated with a relatively high content of lactose $(14 / 100 \mathrm{~g})$. It is very likely that the high lactose content of the diets in the present study favoured the growth of E. coli and L. rhamnosus and thus, colonization by Bifidobacteriaceae and other species of the infant type co-culture was not accomplished. Since Bifidobacteriaceae is expected to be decisive for the metabolic activity of BMO-supplementation, the study did not allow for examination of the anticipated effects. Early studies of humanized mice have shown shifts in the dominant species of microbiota after administration of microbiota from humans [18]. Furthermore, several studies have shown that successful colonization of germ-free mice with a human microbiota is challenging and especially colonization by human co-evolved Bifidobacterium species is not always successful. While mono-association of germ-free mice with the human co-evolved Bifidobacterium bifidum resulted in stable colonization, this strain was outcompeted by murine microbiota, when other mouse co-evolved bacteria were present [19]. The murine microbiota contained Bifidobacterium animalis. Cell assays further showed that $B$. animalis showed stronger adhesion to murine intestinal epithelial cells than to human intestinal cells, which might be the underlying mechanism for outcompeting the B. bifidum strain [19]. Another study by Charbonneau et al. (2016), showed that administration of sialylated BMO to gnotobiotic mice with a Malawian infant type microbiota similarly did not show colonization by Bifidobacterium [7]. The overgrowth of Enterobacteriaceae observed in the current study is probably a result of a low host association of the remaining bacterial strains in the infant type co-culture to the mouse gastrointestinal tract, leading to overgrowth of Enterobacteriaceae. E. coli strains such as the one used in the infant type co-culture are fast growing bacteria with a generation time of $20 \mathrm{~min}$ at optimal conditions [20]. Therefore, it is likely that this strain outcompeted most of the other bacterial strains in the infant co-culture and therefore the possibility of observing any differences between treatment groups was impeded by E. coli overgrowth cecum and colon. The significant $\sim 1.5 \log C F U / g$ higher E. coli numbers identified in cecum content samples from the CON treatment is intriguing. Previous results from our lab showed inhibition of $E$. coli growth in infant type coculture when BMO was present in the media together with lactose compared to media that only contained lactose [9]. The in vitro results further showed higher $B$. longum subsp. longum growth in the treatments with BMO and lactose, while L. rhamnosus growth was not detected. This is in contrast to the present study, where Bifidobacterium were not detected in cecum or fecal samples. These differences between in vitro and in vivo studies might be due to the evolutionary adaptation of Lactobacillus to colonize the gastrointestinal tract of mice. Lactobacilli (family Lactobacilliaceae) are consistently found in high relative abundance in murine microbiota, whereas Bifidobacteria (family Bifidobacteriaceae) are only present in low relative abundance [21]. This suggests that lactobacilli are more likely to colonize the gastrointestinal tract of mice than bifidobacteria, supporting the finding in the present study that Lactobacilliaceae were identified in feces and cecum content, while Bifidobacteriaceae were not. 
Previous studies have shown that cecum content and feces share great similarities in microbial populations, but cecum content shows better separation between treatment groups than end-point fecal samples [22]. Cecum is also the site of the gastrointestinal tract in mice, where the majority of fermentation processes occur [16]. The results from the present study show differences but also similarities in microbiota composition in cecum and fecal samples collected on the day of sacrifice. Specifically, the relative abundance of Lactobacilliaceae appears to be slightly higher in fecal samples, as compared to cecum samples.

In the present study, the metabolic profiles of cecum content and colon content were very similar. Lactose was not detected in the cecum or colon content from mice in either treatment group, indicating that lactose in the diet is either absorbed in the small intestine of the mouse before the dietary bolus reaches the cecum or efficiently fermented by E. coli and L. rhamnosus in the cecum. In contrast, un-metabolized dietary components, such as $3^{\prime}$-SL, was identified in cecum and colon content from samples the BMO treatment. This finding reveals that $3^{\prime}$-SL was not degraded during passage of the upper gastrointestinal tract and reached the lower gastrointestinal tract where it was available for bacterial fermentation. We speculate whether the presence of $3^{\prime} \mathrm{SL}$ and other BMO structures in the cecum and colon of the mice might have had effects in the gastrointestinal tract such as spatial organization of the Enterobacteriaceae, mucus thickness or other parameters related to the gastrointestinal tract, which we did not measure in the present study.

\section{Conclusions}

The present study describes how the application of NMR-based metabolomics in combination with high-throughput 16S rRNA gene amplicon sequencing was useful in comparing metabolic activity, microbiota composition in different compartments of the lower gastrointestinal tract using a gnotobiotic mice model. BMO components were detected in cecum and colon contents, but did not result in microbiota alterations, except for a slightly higher E. coli population in cecum content from CON treatment and slightly higher formate and succinate concentrations in gastrointestinal samples. The unsuccessful colonization by infant type microbiota warrants more studies into how gnotobiotic mouse models can be optimized for studying human microbiota.

Author Contributions: Conceptualization, L.M.A.J., U.K.S., H.J.A., D.S.N., A.K.H. and H.C.B.; Data curation, L.M.A.J., W.K. and J.L.C.M.; Formal analysis, L.M.A.J., H.J.A. and W.K.; Funding acquisition, H.C.B.; Investigation, L.M.A.J., W.K., J.L.C.M. and D.S.N.; Methodology, L.M.A.J., U.K.S., D.S.N., A.K.H. and H.C.B.; Project administration, H.C.B.; Resources, L.M.A.J., H.J.A., W.K., J.L.C.M., D.S.N., A.K.H. and H.C.B.; Software, L.M.A.J. and J.L.C.M.; Supervision, U.K.S., H.J.A., D.S.N., A.K.H. and H.C.B.; Validation, L.M.A.J., U.K.S., H.J.A., W.K., J.L.C.M., D.S.N., A.K.H. and H.C.B.; Visualization, L.M.A.J.; Writing—original draft, L.M.A.J.; Writing—review \& editing, L.M.A.J., U.K.S., H.J.A., W.K., J.L.C.M., D.S.N., A.K.H. and H.C.B. All authors have read and agreed to the published version of the manuscript.

Funding: L.M.A.J. was funded by Arla Foods Amba and Aarhus University as part of L.M.A.J.'s Ph.D. project.

Institutional Review Board Statement: The study was conducted according to the guidelines of the Declaration of Helsinki, and approved by the Animal Experimentation Committee under the Danish Food Administration and was licensed according to the Danish Animal Experimentation Act and the EU Directive 2010/63/EU (License No 2017-15-0201-01262).

Informed Consent Statement: Not applicable.

Data Availability Statement: Data can be released upon request to the corresponding author.

Acknowledgments: L.M.A.J. greatly acknowledges the excellent technical assistance provided by the laboratory staff in A.K.H. and D.S.N.'s research facilities. This work would not have been possible without their important contributions.

Conflicts of Interest: The authors declare no conflict of interest. Arla Food Ingredients Group P/S provided the bovine milk oligosaccharides used in this study. 


\section{References}

1. Sela, D.A.; Mills, D.A. Nursing our microbiota: Molecular linkages between bifidobacteria and milk oligosaccharides. Trends Microbiol. 2010, 18, 298-307. [CrossRef]

2. Newburg, D.S.; Walker, W.A. Protection of the neonate by the innate immune system of developing gut and of human milk. Pediatr. Res. 2007, 61, 2-8. [CrossRef] [PubMed]

3. Akkerman, R.; Faas, M.M.; de Vos, P. Non-digestible carbohydrates in infant formula as substitution for human milk oligosaccharide functions: Effects on microbiota and gut maturation. Crit. Rev. Food Sci. Nutr. 2019, 59, 1486-1497. [CrossRef] [PubMed]

4. Laursen, M.F.; Bahl, M.I.; Michaelsen, K.F.; Licht, T.R. First Foods and Gut Microbes. Front. Microbiol. 2017, 8, 356. [CrossRef]

5. Figueroa-Lozano, S.; Ren, C.; Yin, H.; Pham, H.; van Leeuwen, S.; Dijkhuizen, L.; de Vos, P. The impact of oligosaccharide content, glycosidic linkages and lactose content of galacto-oligosaccharides (GOS) on the expression of mucus-related genes in goblet cells. Food Funct. 2020, 11, 3506-3515. [CrossRef]

6. Ten Bruggencate, S.J.M.; Bovee-Oudenhoven, I.M.J.; Feitsma, A.L.; van Hoffen, E.; Schoterman, M.H.C. Functional role and mechanisms of sialyllactose and other sialylated milk oligosaccharides. Nutr. Rev. 2014, 72, 377-389. [CrossRef] [PubMed]

7. Charbonneau, M.R.; O’Donnell, D.; Blanton, L.V.; Totten, S.M.; Davis, J.C.; Barratt, M.J.; Cheng, J.; Guruge, J.; Talcott, M.; Bain, J.R.; et al. Sialylated milk oligosaccharides promote microbiota-dependent growth in models of infant undernutrition. Cell 2016, 164, 859-871. [CrossRef]

8. Zivkovic, A.M.; Barile, D. Bovine milk as a source of functional oligosaccharides for improving human health. Adv. Nutr. 2011, 2, 284-289. [CrossRef] [PubMed]

9. Jakobsen, L.M.; Sundekilde, U.K.; Andersen, H.J.; Nielsen, D.S.; Bertram, H.C. Lactose and Bovine Milk Oligosaccharides Synergistically Stimulate B. longum subsp. longum Growth in a Simplified Model of the Infant Gut Microbiome. J. Proteome Res. 2019, 18, 3086-3098. [CrossRef]

10. Savorani, F.; Tomasi, G.; Engelsen, S.B. icoshift: A versatile tool for the rapid alignment of 1D NMR spectra. J. Magn. Reson. 2010, 202, 190-202. [CrossRef]

11. Castro-Mejía, J.L.; O’Ferrall, S.; Krych, Ł.; O’Mahony, E.; Namusoke, H.; Lanyero, B.; Kot, W.; Nabukeera-Barungi, N.; Michaelsen, K.F.; Mølgaard, C.; et al. Restitution of gut microbiota in Ugandan children administered with probiotics (Lactobacillus rhamnosus GG and Bifidobacterium animalis subsp. lactis BB-12) during treatment for severe acute malnutrition. Gut Microbes 2020, 11, 855-867. [CrossRef] [PubMed]

12. Krych, L.; Kot, W.; Bendtsen, K.M.; Hansen, A.K.; Vogensen, F.K.; Nielsen, D.S. Have you tried spermine? A rapid and cost-effective method to eliminate dextran sodium sulfate inhibition of PCR and RT-PCR. J. Microbiol. Methods 2018, 144, 1-7. [CrossRef]

13. Edgar, R.C. UNOISE2: Improved error-correction for Illumina 16S and ITS amplicon sequencing. bioRxiv 2016. [CrossRef]

14. McDonald, D.; Price, M.N.; Goodrich, J.; Nawrocki, E.P.; DeSantis, T.Z.; Probst, A.; Andersen, G.L.; Knight, R.; Hugenholtz, P. An improved Greengenes taxonomy with explicit ranks for ecological and evolutionary analyses of bacteria and archaea. ISME J. 2012, 6, 610-618. [CrossRef]

15. Irvin, A.; Cockburn, A.; Primerano, D.A.; Denvir, J.; Boskovic, G.; Infante, A.M.; Wu, G.D.; Cuff, C.F. Diet-Induced Alteration of the Murine Intestinal Microbiome Following Antibiotic Ablation. Microbiology 2017, 7, 545-564. [CrossRef]

16. Nguyen, T.L.A.; Vieira-Silva, S.; Liston, A.; Raes, J. How informative is the mouse for human gut microbiota research? Dis. Models Mech. 2015, 8, 1-16. [CrossRef]

17. Martin, F.-P.J.; Dumas, M.-E.; Wang, Y.; Legido-Quigley, C.; Yap, I.K.; Tang, H.; Zirah, S.; Murphy, G.M.; Cloarec, O.; Lindon, J.C.; et al. A top-down systems biology view of microbiome-mammalian metabolic interactions in a mouse model. Mol. Syst. Biol. 2007, 3, 112. [CrossRef]

18. Kibe, R.; Sakamoto, M.; Yokota, H.; Ishikawa, H.; Aiba, Y.; Koga, Y.; Benno, Y. Movement and fixation of intestinal microbiota after administration of human feces to germfree mice. Appl. Environ. Microbiol. 2005, 71, 3171-3178. [CrossRef]

19. Grimm, V.; Radulovic, K.; Riedel, C.U. Colonization of C57BL/ 6 mice by a potential probiotic Bifidobacterium bifidum strain under germ-free and specific pathogen-free conditions and during experimental colitis. PLoS ONE 2015, 10, e0139935. [CrossRef]

20. Pletnev, P.; Osterman, I.; Sergiev, P.; Bogdanov, A.; Dontsova, O. Survival guide: Escherichia coli in the stationary phase. Acta Naturae 2015, 7, 22-33. [CrossRef] [PubMed]

21. Hugenholtz, F.; de Vos, W.M. Mouse models for human intestinal microbiota research: A critical evaluation. Cell. Mol. Life Sci. 2018, 75, 149-160. [CrossRef] [PubMed]

22. Ericsson, A.C.; Gagliardi, J.; Bouhan, D.; Spollen, W.G.; Givan, S.A.; Franklin, C.L. The influence of caging, bedding, and diet on the composition of the microbiota in different regions of the mouse gut. Sci. Rep. 2018, 8, 1-13. [CrossRef] 